\title{
Treatment Decisions for Newborns at the Threshold of Viability: An Ethical Dilemma
}

\author{
Robin W. Doroshow, MD \\ Joan E. Hodgman, MD \\ Jeffrey J. Pomerance, MD \\ Judith W. Ross, MA \\ Vicki J. Michel, JD \\ Peter M. Luckett, MD \\ Anthony Shaw, MD
}

\begin{abstract}
The major ethical issues involved in decision-making in the care of extremely low birth weight newborns are analyzed here. We propose a schema for assessment and management of these infants that is consistent with ethical principles broadly accepted by the pediatric community, and which takes into account mortality risk at any given institution rather than arbitrary weight limits, with a major decisionmaking role for the infant's parents. When possible, the decision of whether or not to resuscitate should be made before delivery; when not possible, delivery room resuscitation is recommended, and the decision to continue or withdraw care should be made subsequently based on likelihood of survival and the wishes of the parents.
\end{abstract}

Journal of Perinatology 2000; 20:379-383.

The definition of "viable" continues to change with current perinatal care as ever smaller and less mature newborns survive. The recent literature defines the lower limit of viability as 22 weeks' gestation, with essentially no chance of survival at or below this maturity. ${ }^{1,2}$ Current survival statistics improve dramatically with each additional intrauterine week to better than $50 \%$ at 24 weeks gestation and over $70 \%$ at 26 weeks' gestation. These early gestations usually correspond to birth weights of $\leq 750 \mathrm{gm}$. Expecta-

\footnotetext{
Department of Pediatrics (R. W. D.), Harbor-University of California Los Angeles Medical Center, Torrance, CA; Department of Pediatrics (J. E. H.), Los Angeles County-University of Southern California Medical Center, Los Angeles, CA; Department of Pediatrics (J.J. P.), Cedars-Sinai Medical Center, Los Angeles, CA; Center for Healthcare Ethics (J. W. R.), Orange, CA; University of Southern California Law Center (V.J.M.), Los Angeles, CA; Department of Pediatrics (P. M. L.), University of Texas, Southwestern Medical Center, Dallas, TX; and Department of Surgery, Olive View Medical Center, Sylmar, CA.
}

This work was supported by Chapter 2, District IX, American Academy of Pediatrics.

The deliberations on which this paper is based were conducted by the authors as members of the Committee on Bioethics of Chapter 2, District IX of the American Academy of Pediatrics. The recommendations contained herein do not represent those of the American Academy of Pediatrics.

Address correspondence and reprint requests to Robin W. Doroshow, MD, Long Beach Memorial Heart Institute, 2801 Atlantic Avenue, Long Beach, CA 90801. tions for survival by birth weight are negligible for infants of $<500$ gm, increase to better than $30 \%$ for infants of 500 - to 750 -gm birth weight, and reach over $70 \%$ for infants weighing $>750$ gm at birth. ${ }^{3,4}$ It is important to note that results are highly variable across centers, with some reporting survival rates of $>50 \%$ in the 500 - to 750 -gm group. ${ }^{4}$

At the lower limits, not only is the likelihood of survival small but that of intact survival is even lower, ${ }^{5}$ while the emotional, human energy, and financial costs are very high. In some cases, parents prefer not to be aggressive; in others, they wish the physicians to "do everything possible" despite little or no probability of success.

Physicians must make recommendations and decisions, but what are they to base them on? Management decisions often must be made quickly, with little opportunity for detailed evaluation of the infant, or for discussion and reflection. An informal survey of local hospitals revealed substantially varying approaches, and no formal policies. The medical literature reflects this variation. ${ }^{2,6-9}$ For example, in some centers, resuscitation is attempted for all liveborn infants. In others, all infants within a defined category (e.g., at least 23 weeks' gestation) are routinely resuscitated; in yet others, the physician attending the delivery decides whether to attempt resuscitation. ${ }^{10}$ Practicing pediatricians and neonatologists may be unaware of which approach is endorsed in their hospitals, or the appropriate policy may be incomplete, unclear, or in conflict with recent legal decisions. ${ }^{11}$ The existence of such variations led this group to propose the following principles and procedures to make it more likely that similar infants would be treated similarly, and to clarify parental and physician roles in decision-making for these very vulnerable infants.

\section{ETHICAL PRINCIPLES ${ }^{12}$}

The principle of beneficence (doing good) is the major force behind the efforts to rescue these newborns. Society has traditionally seen medicine's ability to prolong life as an unqualified good, but in more recent years, new technological capacities have led society to question whether some uses of medical technology constitute prolonging death rather than prolonging life. Furthermore, when society permits forgoing treatment for patients with serious diseases or those in a persistent vegetative state, it is acknowledging that life is not always preferable to death, particularly if continued life involves suffering that is very burdensome. ${ }^{13}$

Physicians are also bound by the principle of nonmaleficence (doing no harm). The very premature infant risks death and long- 
term handicap. The infant and family also experience significant suffering in the course of the infant's care. Physicians routinely inflict such suffering on patients and their families. This is justified by the expected outcome (i.e., that the benefits will exceed the burdens). However, when the likelihood of survival becomes extremely small, inflicting such suffering may become unjustifiable. For example, when there is a $10 \%$ chance of survival, out of 10 such babies, all 10 will experience the burden of our ministrations while only one will benefit. Unfortunately, we cannot accurately predict which one that will be.

The principle of autonomy (respecting individual preferences) does not apply to these decisions, as infants have no autonomy. Parents do have a moral right and a legal duty to make treatment decisions in the best interests of their child. Physicians too have a moral right and legal duty to act in the best interests of their infant patients. Because a best-interests judgment is based on a variety of factors and on uncertain medical outcomes, and because physicians and parents may have different values, parents and physicians may disagree about what action serves the infant's best interests; however, each has an obligation to make that judgment. ${ }^{14}$

The principle of justice applies not only to treating similar infants similarly (fairness), but also to the effective use of resources (distributive justice). In an era of rapidly rising medical costs and limited access to specialized care such as neonatal intensive care, aggressive care of patients with a remote possibility of intact survival may be an inappropriate way to allocate resources. Our society is only recently, and reluctantly, looking at this issue in relation to other values such as autonomy and beneficence. Its earliest applications are likely to be situations with high cost and low chance of success, as in the case of extreme prematurity. A tiered system of health care, which exists in the United States at present, inherently leads to unfairness. Further limitation of resources simply makes this more apparent. However, this unfairness would only be intensified if allocation decisions were made relative to individual patients rather than at a policy level..$^{15}$

\section{LEGAL ISSUES}

In 1984, the American Academy of Pediatrics issued a policy statement, "Principles of Treatment of Disabled Infants,"16 in response to a proposed federal statute and regulations generally known as the Baby Doe regulations. Both documents clearly stated that actual or anticipated disability must not be the basis for withholding medical care. However, these policies also stated that there was no justification for futile treatment that prolonged dying. A national discussion arose in the context of denying care to newborn infants with congenital defects with a certain prognosis for mental retardation such as Down syndrome.

The premature infant at the threshold of viability differs significantly from the infant with congenital defects. The expected outcomes for survivors of extreme prematurity span the entire range from severe handicap to normal, and prognosticating whether individual infants will in fact survive, and if so their degree of handicap, is fraught with greater uncertainty than in the case of congenital defects such as those associated with Down syndrome.

In the more than 10 years since the Baby Doe regulations were approved, public sentiment has been changing. Providing treatment of doubtful value regardless of cost, which was strongly recommended in the original policies, is increasingly coming under question. ${ }^{17}$ Enforcement of the regulations depends on state laws, and a state legal standard is emerging that is flexible and allows a quality of life analysis because it is based on parental authority. ${ }^{918}$ Cost-benefit analysis is being applied to what is technologically possible and what is statistically probable. Recent case law has confirmed that there are situations in which a treatment is clearly not mandatory, as in State $v$ Messenger ${ }^{11}$ and even those in which it might constitute battery if the parents decline treatment. ${ }^{9}$

\section{RECOMMENDATIONS}

After considerable deliberation, the American Academy of Pediatrics California Chapter 2 Bioethics Committee has developed a set of recommendations for the management of infants weighing $<750$ gm at birth or with a gestational age of $<25$ weeks; we hope these guidelines will serve as a starting point for open discussion in the pediatric community and in particular among representatives of hospitals that provide high-level neonatal care. Similar guidelines have been in effect at some of our own institutions. Although a uniform set of guidelines may not be reached, open discussion should facilitate the development of clearer policies within institutions.

\section{Data Availability}

Caregivers should gather as much data as possible on the outcome probabilities of similar infants. The appropriate department (Pediatrics, Obstetrics, Neonatology, or Perinatology) should keep, and make readily available, up-to-date statistics with regard to survival and major disability in very small premature infants cared for at that institution and elsewhere in the region. These data must then be presented to the parents in a manner understandable to them, as soon as possible. If this occurs before delivery, it may affect not only management of the infant, but also obstetrical management, such as the option for emergency cesarean delivery in cases of fetal distress. If the survival statistics in the specific hospital are nil because aggressive care had not previously been given infants of that weight, the parents must be so informed. Physicians should offer a recommendation to the parents based on these data.

\section{Anticipating Decisions}

Whenever possible, discussion regarding resuscitation should be initiated before delivery. Both obstetrician and pediatrician should participate in this discussion with the parents. At minimum, all parties need to know and accept the parents' decision to avoid errors based on lack of communication. ${ }^{11}$ Thus, when labor is very premature in onset, the parents should be provided with pertinent information, and a plan formulated. However, this plan must take into account the inaccuracy of predicting gestational age and weight, and therefore everyone should be prepared to alter decisions in the face of new information. 


\section{Resuscitation}

Delivery room decisions regarding resuscitation are complicated by multiple factors. (1) Although there may be advance knowledge of the impending dilemma, often there is very little time for deliberation.

(2) Parents' decision-making ability may be compromised by physi-

cal and emotional stress. (3) Gestational age is very important in predicting outcome, but often difficult to assess in the very premature infant. Obstetric input may improve accuracy, particularly if there has been early ultrasound evaluation for the purpose of gestational assessment. In contrast, birth weight, although it cannot be accurately predicted antenatally, can be measured accurately and is the best objective independent variable in predicting outcome. For this reason, most mortality and morbidity data, both published and local, are related to birth weight rather than gestational age. (4) The current survival figures for a given institution may be unknown to the attending physician at the time of delivery. (5) The chance of survival without immediate intervention is small.

For these reasons, we recommend that delivery room resuscitation be attempted in all infants for whom it is technologically feasible, except where a previous discussion has led to the decision not to resuscitate or where the physician determines that the infant has a lethal congenital anomaly or close to $100 \%$ mortality risk. This does not mandate continued aggressive care of these patients, but defers the decision, enabling the parties involved to collect the needed information and discuss the issues. This approach assumes that withholding care and withdrawing care are ethically equivalent, a premise which we accept. ${ }^{13,19}$ The physician must accept it not only in theory, but in fact, and be willing to withdraw medical support after a brief period (perhaps within the first 48 hours) of stabilization, discussion, and reflection.

\section{Postresuscitation Care}

We propose the following schema of clinical categories of extremely low birth weight newborns (see Appendix):

Category A: Infants with a very high risk of mortality despite all efforts. The definition of a "very high mortality risk" will require much discussion, but mortality rates in excess of $75 \%$ seem appropriate.

Category B: Infants with a high mortality risk (for example, between $50 \%$ and $75 \%$ ).

Category C: Infants with a moderate mortality risk (for example, $<50 \%$ ).

Treatment regimens can be broadly divided into two levels:

Basic care. The goal of this category is humane care for a baby who has very little probability of survival. This category of care would include warmth, comfort, and fluids. The infant who is obviously not sustaining spontaneous respiration would receive warmth and comfort only. For the infant who is breathing spontaneously, intravenous fluids and supplemental oxygen should be added. This will help ensure that the infant who is more vital than expected does not survive with deficits resulting from withholding of basic care.
Advanced care. The goal of this category is the provision of medically indicated treatment for a baby who has a reasonable probability of survival. In addition to the basic care to which all infants are entitled, this treatment category includes the administration of medications, surgical intervention, and life support such as mechanical ventilation and pressor agents.

Infants judged by the physician to be nonviable, but who survive beyond the delivery room despite the decision not to intervene, should receive basic care. If parents wish more aggressive care, basic care is continued while further discussions are held with the parents and the infant's condition is reassessed.

If the parents request more aggressive support for their infant, advanced care should be provided. Until such time as society decides to withhold support for selected patient populations or conditions, treatment should be provided, regardless of physician recommendation, if parents wish it after receiving adequate information and physician recommendations.

Physicians should help parents to understand the nature of the uncertain benefits and risks and the certain burdens of treatment for babies in category $B$ and accept their informed decision either to provide basic care or to provide advanced care. Physicians may wish to make a recommendation but should acknowledge that either decision can be morally supported.

Physicians should recommend and routinely initiate advanced care for babies in category $C$ because such treatment is clearly in the infant's best interests even if the parents disagree. If parents disagree with the provision of advanced care, communication with them should continue in an effort to explain the reasoning behind the decision while advanced care is being provided. In addition, other resources for resolving conflict should be used. Examples include re-evaluation and further medical consultation to ensure assignment of the infant to the appropriate clinical category, consultation with clergy, transfer of care of the infant to another physician or facility, and bioethics committee consultation. ${ }^{20}$ Very rarely, court approval may need to be sought for selected infants.

Lines of communication must be kept open to discuss the implications of a treatment decision, and every attempt should be made to achieve a solution that is acceptable to all major parties. Frequent reassessment is indicated for patients in both treatment categories, with the understanding that revisions in the treatment plan may be appropriate.

\section{Parental Input}

Under ordinary circumstances, parents are likely to be the best advocates for their infants. ${ }^{21}$ Therefore, parental wishes should generally be followed. Current American opinion appears to be divided over whether perceived future quality of life should be a factor in deciding appropriateness of treatment. ${ }^{22}$ We believe parents, as the best advocates for their child, should consider those issues that are important to them.

\section{Additional Resources}

The individual physician should serve as a provider of medical care and also as one of a variety of social resources available to 
the parents. Making the best possible decisions in the difficult situations addressed here is challenging to the professional health care provider; it may be overwhelming to the parent. The individual physician is in a position to be of great help through timely, compassionate, and knowledgeable application of the art of medicine. He or she will doubtless wish to involve additional physicians and other health care professionals and counselors in providing support to the parents. Ethics committees and multidisciplinary clinical groups may also be helpful as a forum for discussion of these cases. ${ }^{20,22}$

\section{DISCUSSION}

We have attempted to assess the principles and issues relevant to this medical and ethical dilemma, using them to develop a set of guidelines that proposes a strategy for decision-making. This strategy takes into account the known medical facts including in particular the degree of uncertainty of outcome for any individual infant. Our approach incorporates elements of a "statistical prognostic strategy" as defined by Rhoden ${ }^{10}$ relying on predictions of outcome based on birth weight. However, that strategy, when applied exclusively, does not allow for significant parental input or for the addition of individualized prognostic data.

To correct for these shortcomings, we have added elements of Rhoden's "individualized prognostic strategy" in proposing a major role for the parents, particularly when the prognosis is neither good nor very poor. In this respect, an important feature of our guidelines is the recommendation, when in doubt, to resuscitate in the delivery room and to reassess later, with willingness to withdraw support in the neonatal intensive care unit. This reassessment should incorporate features specific to the infant, such as accurate assessment of weight, estimation of gestational age, response to treatment, and discovery of congenital anomalies, and will yield data essential to both the medical team and the family in coping with this difficult situation. We hope that these modifications of the statistical prognostic strategy will minimize the potential both for overtreatment and for undertreatment of an extremely premature infant while taking into account the parental interpretation of their child's best interests.

We believe that the application of this type of strategy would give much-needed support to individual physicians, especially house officers and others less experienced in making these decisions. It would promote a degree of uniformity, not in the actual decisions themselves, but in the approach to these decisions, that reflects the ethical principles held by our society. We have chosen not to stress a specific cutoff weight or gestational age, as we are acutely aware that medical advances continue to push back the threshold of viability.

Several efforts on the part of leading medical organizations to address this problem have been published recently. The Committee on the Fetus and Newborn of the American Academy of Pediatrics ${ }^{19}$ outlined the relevant fundamental principles, with which we are in agreement, including the importance of an active role for the parents, the use of a projected outcome, and the moral equality of withdrawing treatment and withholding it. Their statement did not, however, delineate an actual strategy that applies these points. It also implied a larger role for the physician in decision-making than we have recommended here.

The same committee, in conjunction with the Committee on Obstetric Practice of the American College of Obstetricians and Gynecologists, shortly thereafter published a statement focusing on prenatal management of extreme prematurity. ${ }^{23}$ Although this policy proposes more concrete strategies for proceeding, it does not define the role of the parents. It also fails to distinguish between initial resuscitation and postresuscitation neonatal care. By placing heavy emphasis on delivery room management, it puts unnecessary reliance on estimated gestational age and (sometimes) rapid decision-making, and minimizes the role of individual assessment and prognosis.

This is also the major shortcoming in the guidelines proposed by the Fetus and Newborn Committee of the Canadian Pediatric Society and the Maternal-Fetal Medicine Committee of the Society of Obstetricians and Gynecologists of Canada, ${ }^{2}$ which stress the importance of making "a decision to withhold or withdraw treatment immediately after birth." In other respects, however, we are in strong agreement with their guidelines, which apply the statistical prognostic strategy by dividing the infants into categories according to mortality risk. They also emphasize short-term and long-term outcomes at the institution involved, rather than aggregate results published in the medical literature.

There is little doubt that in this era of managed care, the cost efficiency of neonatal intensive care will be evaluated. If we value distributive justice, and our culture is now in the process of rapidly increasing the worth it places on that principle, assessment of outcome in light of cost may be used as a reflection of the value of guidelines such as these. The cost of overtreatment is of major concern and easy to measure, in dollars per intact survivor. The additional cost of long-term care for impaired survivors must be taken into account, but more difficult to appraise is the cost of the death of the infant who might have been a survivor.

\section{SUMMARY}

Treatment decisions in the management of infants at the threshold of viability are faced commonly, but not often discussed openly. Such decisions may be very difficult for both physicians and parents. We have analyzed the major ethical issues involved and proposed a schema for assessment and management that is consistent with ethical principles broadly accepted by the pediatric community ${ }^{6,19}$ and which takes into account mortality risk at any given institution rather than arbitrary weight limits, with a major decision-making role for the infant's parents. 


\section{Appendix: Clinical Categories of Extremely Low Birth Weight Infants}

I. Nonviable (lethal congenital defect or close to $100 \%$ risk of mortality): Resuscitation not indicated, but if infant survives delivery, physician initiates basic care.

If parents wish more aggressive care, basic care is continued while further discussions are held with parents.

II. Possibly viable: Postresuscitation care

Category A: Borderline viable (very high risk of mortality):

Physician initiates basic care.

If parents wish more aggressive care, advanced care is provided.

Category B: Limited viability (high risk of mortality):

Physician recommends parental discretion.

If parents want no treatment, basic care is given.

If parents want treatment, advanced care is given.

Category C: Potentially viable (moderate risk of mortality):

Physician initiates advanced care.

If parents agree, advanced care is continued.

If parents do not want treatment, advanced care is continued while alternatives are explored.

\section{References}

1. Allen MC, Donohue PK, Dusman AE. The limit of viability: neonatal outcome of infants born at 22 to 25 weeks' gestation. N Engl J Med 1993;329:1597-601.

2. Canadian Paediatric Society, Society of Obstetricians, and Gynaecologists of Canada. Management of the woman with threatened birth of an infant of extremely low gestational age. Can Med Assoc J 1994;151:547-53.

3. Hack M, Wright LL, Shankaran S, et al. Very-low-birth-weight outcomes of the National Institute of Child Health and Human Development Neonatal Network, November 1989 to October 1990. Am J Obstet Gynecol 1995:172:457-64.

4. Ehrenhaft PM, Wagner JL, Herdman RC. Changing prognosis for very low birth weight infants. Obstet Gynecol 1989;74:528-35.

5. Escobar GJ, Lindenberg B, Pettiti DB. Outcome among surviving very low birth weight infants: a meta-analysis. Arch Dis Child 1991;66:204-11.

6. Sanders MR, Donohue PK, Oberdorf MA, et al. Perceptions of the limit of viabil- ity: neonatologists' attitudes toward extremely preterm infants. J Perinatol 1995; 15:494-502.

7. Carter BS. Neonatologists and bioethics after Baby Doe. J Perinatol 1993;13:14450 .

8. Kopelman LM, Irons TG, Kopelman AE. Neonatologists judge the "Baby Doe" regulations. N Engl J Med 1988;318:677-83.

9. Tyson J. Evidence-based ethics and the care of premature infants. Future Child 1995;5:197-213.

10. Rhoden NK. Treating Baby Doe. the ethics of uncertainty. Hastings Cent Rep 1986;24:34-42.

11. Clark FI. Making sense of State v Messenger. Pediatrics 1996;97:579-83.

12. Johnson AR, Seigler M, Winslade WJ. Clinical Ethics, 3rd ed. New York: McGrawHill; 1992.

13. President's Commission for the Study of Ethical Problems in Medicine and Biomedical and Behavioral Research. Deciding to Forego Life-Sustaining Treatment. Washington, DC: U.S. Government Printing Office; 1983:207-15.

14. Lee SK, Penner PL, Cox M. Comparison of the attitudes of healthcare professionals and parents toward active treatment of very low birth weight infants. Pediatrics 1991;88:110-4.

15. Ellis RS III. Letting defective babies die: who decides? Am J Law Med 1982;7:393-423.

16. American Academy of Pediatrics Joint Policy Statement. Principles of treatment of disabled infants. Pediatrics 1984;73:559-60.

17. Doyle LW, Murton LJ, Kitchen WH. Increasing the survival of extremely immature (24- to 28-weeks' gestation) infants: at what cost? Med J Aust 1989;150:558-68.

18. Clark FI. Intensive care treatment decisions: the roots of our confusion. Pediatrics 1994;94:98-101.

19. American Academy of Pediatrics. The initiation or withdrawal of treatment for high-risk newborns. Pediatrics 1995;96:362-36.

20. Ross JW, Bailey C, Michel V, Pugh D. Handbook for Hospital Ethics Committees. Chicago: American Hospital Publishing; 1986.

21. Harrison H. The principles for family-centered neonatal care. Pediatrics 1993;92: 643-50.

22. Campbell AGM, McHaffie HE. Prolonging life and allowing death: infants. J Med Ethics 1995;21:339-44.

23. American Academy of Pediatrics, American College of Obstetricians and Gynecologists. Perinatal care at the threshold of viability. Pediatrics 1995; 96:974-6. 